\title{
Fast Tonotopy Mapping of the Rat Auditory Cortex With a Custom-Made Electrode Array
}

\author{
J. LINDOVSKÝ ${ }^{1}$, K. PYSANENKO ${ }^{1}$, J. POPELÁR̆ ${ }^{1}$, J. SYKA ${ }^{1}$ \\ ${ }^{1}$ Institute of Experimental Medicine of the Czech Academy of Sciences, Prague, Czech Republic
}

Received December 12, 2017

Accepted June 12, 2018

On-line September 11, 2018

\begin{abstract}
Summary
We present a custom-made multielectrode array for the recording of evoked potentials during acute experiments in rats, which offers a quick and reliable estimation of the cortical tonotopy. The array consists of electrodes represented by insulated copper wires of $0.09 \mathrm{~mm}$ diameter fixed in epoxy resin in a $3 \times 5$ arrangement, with final impedances of $410-800 \mathrm{kOhm}$. The array was placed on the brain surface of anesthetized rats approximately at the location of the auditory cortex (AC) and the cortical evoked potentials (middle-latency responses, MLR) were elicited by a series of tone pips of different frequencies at $50 \mathrm{~dB}$ of sound pressure level (SPL) intensity. The frequency that evoked the highest MLR amplitude (best frequency, BF) was identified for each electrode. The obtained distribution of the BFs characterized the cortical tonotopy, and it correlated with the frequency selectivity of neurons recorded at the same positions by an extracellular microelectrode. Although the space resolution of the array did not allow for the identification of $A C$ sub regions, the array proved to be a reliable tool for a quick estimation and prediction of areas of interest for the subsequent measurements of neurons by more precise techniques.
\end{abstract}

\section{Key words}

Electrode array - Auditory cortex • Rat - Middle-latency responses $\bullet$ Multiple-unit activity

\section{Corresponding author}

J. Lindovský, Department of Auditory Neuroscience, Institute of Experimental Medicine of the Czech Academy of Sciences, Vídeňská 1083, 14220 Prague, Czech Republic. E-mail: jiri.lindovsky@img.cas.cz
The main criterion for the functional parcellation of the AC is its tonotopy, which is a systematic spatial arrangement of neurons tuned to individual sound frequencies. According to the original study by Sally and Kelly (1988), the primary auditory field in the rat (AI) was characterized by its tonotopic gradient with high frequencies represented rostrally, and low frequencies caudally. In subsequent electrophysiological investigations, this basic finding was confirmed (Doron et al. 2002, Kalatsky et al. 2005). In addition to the AI, other adjacent auditory cortical areas were described in rats and other rodents as an anterior auditory field (AAF), the posterior auditory field (PAF), and the suprarhinal auditory field (SRAF) (Thomas and Tillein 1997, Rutkowski et al. 2003, Pandya et al. 2008, Profant et al. 2013).

In electrophysiological recordings with microelectrodes, the basal orientation for the placement of the electrode in AC is usually made with respect to the vascular architecture. However, in our experience the variability of its pattern among animals is vast, and in many cases the inserted electrode failed to register any response to sound stimulation. Explanations for such recordings could be either the pathological state of the $\mathrm{AC}$, or false electrode positioning, i.e. outside the AC. To minimize the risk of incorrectly positioned microelectrodes, we designed a surface electrode array, which allows for a fast and reliable estimation of the AI tonotopy and determination of the AI boundary in the rat cortex.

The array consisted of 15 insulated copper wires of $0.09 \mathrm{~mm}$ diameter, arranged in a matrix of $5 \times 3$ 
with the overall size of approximately $2 \times 1 \mathrm{~mm}$ (Fig. 1A). The fabrication of the array was completed in a few simple steps. First, the electrode layout was printed on a thin plastic film, which was put on a piece of modeling clay and pierced with a sharp needle under the microscope in the positions of the future electrodes. The wires were inserted in the created pinholes and fixed with epoxy resin. After curing the plastic film was removed, the resin was flattened and smoothed using very fine sandpaper and the opposite ends of all 15 wires were soldered to a 16-pin connector (dual-in-line, DIL 16). The 16th pin was reserved for a ground electrode. Finally, individual electrodes were checked for impedance, which varied between $410-800 \mathrm{kOhm}$. This complete process of array fabrication takes approximately $24 \mathrm{~h}$, including the epoxy resin curing time.

The data presented here were obtained in eight Long-Evans rats aged 3-4 months. The use of animals was approved by the Ethics Committee of the Institute of Experimental Medicine of the Czech Academy of Sciences, and followed the guidelines of the EU Directive 2010/63/EU for animal experiments.
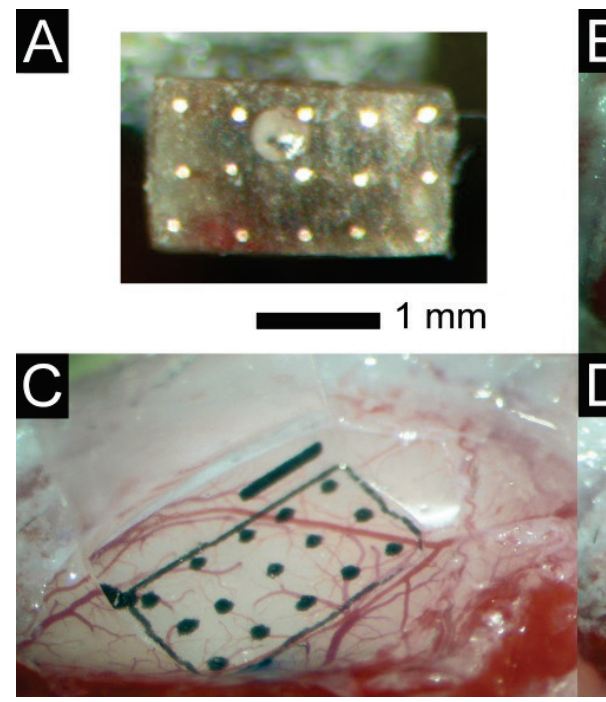

The rats were anesthetized with an intramuscular injection of a mixture of $35 \mathrm{mg} / \mathrm{kg}$ ketamine (Calypsol $50 \mathrm{mg} / \mathrm{ml}$; Gedeon Richter, Budapest, Hungary) and $6 \mathrm{mg} / \mathrm{kg}$ xylazine (Rometar $20 \mathrm{mg} / \mathrm{ml}$; Bioveta, Ivanovice na Hane, Czech Republic). The craniotomy in the right temporal region was performed between the temporoparietal suture and linea temporalis. After removal of the dura mater (Fig. 1B), a transparent sham array with printed marks in places of electrodes was placed in the opening, gently touching the cortex surface, and photographed at 200x magnification (Fig. 1C). As the sham array was transparent, the pattern of blood vessels below was clearly visible. The sham array was placed and photographed in 1-3 locations to cover the entire surface of the craniotomy. These pictures subsequently served as a reference for the orientation of the real array placement (Fig. 1D) and for the reconstruction of AC tonotopical maps. MLRs were recorded in each location. MLRs were elicited by a series of tones of $60 \mathrm{~ms}$ duration in the frequency range of 1 to $45 \mathrm{kHz}$ with 2 /octave step, a stimulation rate was $1.3 \mathrm{~Hz}$ and the responses were
Fig. 1. Electrode array placement. A) The electrode array, its surface with individual electrodes seen from the bottom. B) Craniotomy after removal of the dura mater. C) A transparent sham electrode with photocopied outlines of the real electrode array and the cortical vasculature visible underneath. D) The array placed at the same position as the sham electrode according to the vasculature patterns. calculated as an average of 15 repetitions. A sound intensity of $50 \mathrm{~dB}$ SPL was chosen after a series of optimization tests as a compromise between being too low (evoked small MLR amplitudes with poor signal-tonoise ratio) and too high (large responses but poor frequency tuning). The stimuli were generated and the MLR was analyzed by TDT hardware (Tucker-Davis Technologies System III, Alachua, USA) using Brainware software in 'local field' mode. Stimuli were presented in free-field conditions via ribbon tweeter RAAL 70-20XR and mid-bass woofer Selenium 6W4P, placed $70 \mathrm{~cm}$ in front of the animal's head. In the recorded MLR waveform the first positive peak P1, and the first negative peak N1 (Fig. 2A) were automatically detected (own script in Matlab, MathWorks) and the P1-N1 amplitude difference was defined as the amplitude of MLR. The frequency of the stimulus that evoked the highest MLR amplitude at $50 \mathrm{~dB}$ SPL stimulation intensity in a given position was taken as the local best frequency (BF). Finally, using our own JavaScript program, the results were plotted into a photograph of the 
cortex as dots. The BFs were color-coded, the diameter of the dots corresponded to the MLR amplitudes and their positions were assessed from the sham electrode photographs taken earlier. The locations where MLR amplitude did not exceed $100 \mu \mathrm{V}$ were regarded as non-responsive to tonal stimulation and were depicted as crosses. Experiments were performed in a soundattenuated anechoic room. During MLR recordings, the body temperature of the animal was maintained at $37-38^{\circ} \mathrm{C}$ using an electrically controlled heating pad.
A
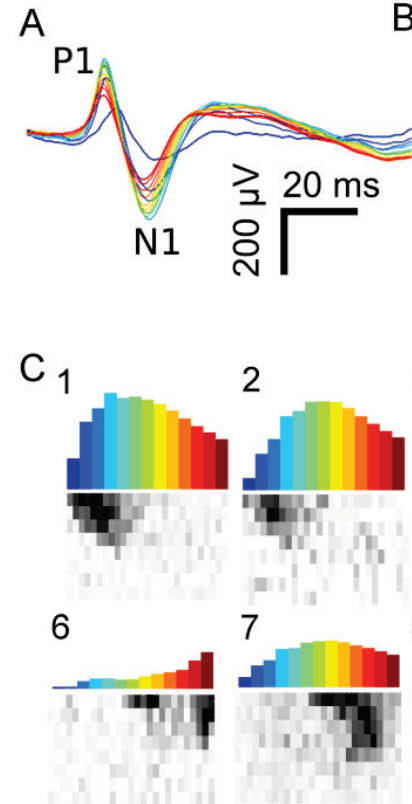

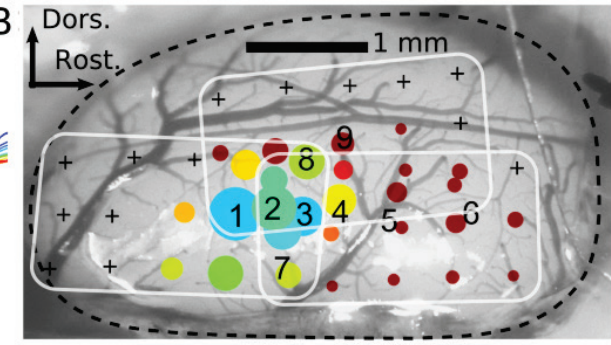

3
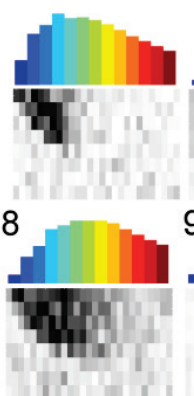

4
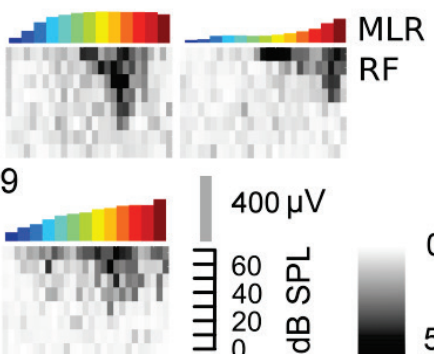

5
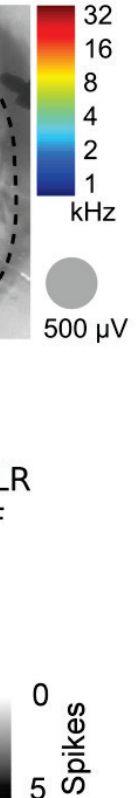

Fig. 2. Middle latency responses and neuronal properties in one example animal. A) Example waveforms of MLR evoked by tones of constant intensity (50 dB SPL) and various frequencies (1-45 kHz, consult B for color-coding). Amplitude difference between the first positive and the first negative peak (P1-N1) was considered as the amplitude of MLR. All responses shown here were recorded at the same cortical position (\#1 in B and C). B) Photograph of the rat cortex with the craniotomy border (dashed line) and electrode positions (dots and crosses). The color of the dots represents the frequency of stimulation which evoked the highest amplitude of MLR (best frequency, BF); the value of that amplitude is represented by the dot diameter. Locations where MLR amplitudes did not reach $100 \mu \mathrm{V}$ were not analyzed due to low signal-to-noise ratio and are represented as crosses. MLR amplitudes obtained in the locations 1-9 are plotted as bar graphs in C. Placing the array successively in three different positions

(white outlines) was necessary to cover the entire area. Note that the main tuning of the MLR rises from low to high frequencies approximately caudo-rostrally, but there were some middle-frequency responses (BF around $8 \mathrm{kHz}$, yellow-green dots) also found in the most caudal positions. C) MLR amplitudes represented as bar graphs, the numbers correspond to MLR recording sites in B. The grey plots below the MLR responses indicate multi-unit responses recorded at corresponding sites (same frequency axes for MLRs and multiunit responses).
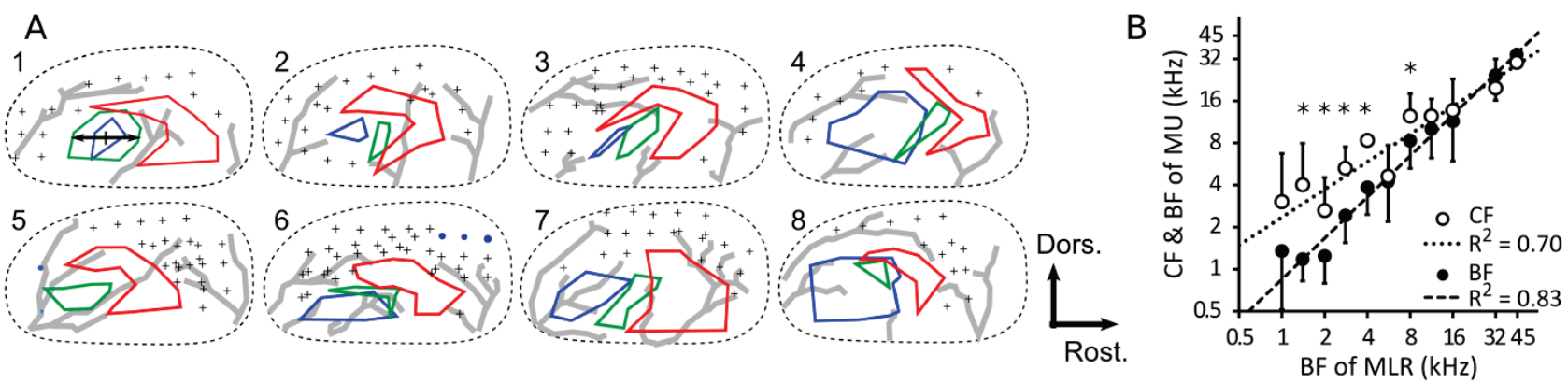

Fig. 3. MLR maps and neuronal properties in all eight animals. A) A simplified map of tonotopy based on the MLR analyzed for each animal (animal 1 is the same as in Figure 2). The borders delineate areas where the BFs of MLR were of low $(<4 \mathrm{kHz}$, blue line), middle (4-16 kHz, green line), or high ( $>16 \mathrm{kHz}$, red line) frequency, respectively. The crosses stand for locations where the MLR amplitude did not reach $100 \mu \mathrm{V}$. Note the striking interindividual differences in position and size of the areas. Arrows in map 1 symbolize a reversal of the main tonotopical order found in the caudal direction. According to our observations, the pattern of the main blood vessels (grey lines) does not provide any helpful information related to the cortical tonotopy. B) Comparison of MLR tuning with tuning of neurons. Five to eight points (out of 30-45 present in a typical complete map) were selected in each animal that had the highest MLR amplitude and the highest frequency specificity (response to BF in comparison with response to other frequencies). One to three multiple-unit recordings in each position were recorded and their best frequency (BF, the frequency resulting in the most intensive firing) at $50 \mathrm{~dB}$ SPL intensity, as well as characteristic frequency (CF, frequency of the weakest stimulus that evoked a response) was determined. For each $B F$ of MLR the average of neuronal $B F-S D$ and $C F+S D$ is plotted. Lines represent a linear regression of the data with corresponding R-squared being 0.70 (CF) and $0.83(\mathrm{BF})$, respectively. Asterisks denote statistical difference between neuronal CF and neuronal BF (paired t-test, $\mathrm{p}<0.05$ ). 
The distribution of BFs of MLRs on the cortical surface roughly corresponded to the known tonotopy of the primary auditory cortex (AI), i.e. low frequency-tuned MLRs predominated in ventro-caudal regions, higher BFs gradually appeared rostro-dorsally. The recorded locations were divided into 3 groups according to the BF: low-frequency places ( $\mathrm{L}, \mathrm{BF}<4 \mathrm{kHz}$ ), middle-frequency places $(\mathrm{M}, \mathrm{BF} 4-16 \mathrm{kHz})$ and high-frequency places $(\mathrm{H}, \mathrm{BF}>16 \mathrm{kHz})$. Among these three groups, a significant decrease was observed in amplitudes (ANOVA, $\mathrm{p}<0.001$ ) as well as a shortening of the $\mathrm{P} 1$ and $\mathrm{N} 1$ latencies (ANOVA, $\mathrm{p}<0.001$ ) dependent on BF. Amplitudes (average \pm SD): $323 \pm 211 \mu \mathrm{V}(\mathrm{L}), 251 \pm 98 \mu \mathrm{V}(\mathrm{M})$ and $166 \pm 64 \mu \mathrm{V}$ (H). P1 latency: $21.0 \pm 3.1 \mathrm{~ms}(\mathrm{~L}), 20.6 \pm 2.0 \mathrm{~ms}$ (M), $19.2 \pm 1.6 \mathrm{~ms}(\mathrm{H}) . \mathrm{N} 1$ latency: $36.2 \pm 3.8 \mathrm{~ms}$ (L), $34.8 \pm 3.0 \mathrm{~ms}(\mathrm{M}), 32.8 \pm 1.6 \mathrm{~ms}(\mathrm{H})$. Areas containing positions with $\mathrm{L}, \mathrm{M}$ and $\mathrm{H}$ characteristics are compared in Figure $3 \mathrm{~A}$ for all animals. It is notable how much the position and size of the areas differed among individuals. For example, the comparison of the large $\mathrm{L}$ area of animals 4 and 8 to the dimension of the same area in animal 3. Additionally, in animal 5, only two individual isolated points were found to have $\mathrm{L}$ characteristics.

Apart from AI, there are several other well-known tonotopically organized regions in the vicinity of AI (Rutkowski et al. 2003, Profant et al. 2013). Unfortunately, no reliable evidence of these secondary auditory fields could be found by MLR analysis in our experiments, although in principle, evoked potentials here can be detected (Ohl et al. 2000, Takahashi et al. 2005). Since these regions are substantially smaller than AI and their dimensions are comparable to the inter-electrode distance of our array, we cannot exclude that they might have remained below the spatial resolution of our mapping. On the other hand, in a unique case we saw distinct low-frequency-tuned MLRs frontally (Fig. 3A, animal 6, blue dots) and a reversal of the tonotopical order caudally (Fig. 3A, animal 1, arrows) which might represent responses of some of these secondary auditory fields.

To verify the ability of predicting cortical tonotopy from MLR data, the extracellular activity of individual neurons was recorded in selected locations of the AC. Five to eight points of interest were selected in the MLR map of each animal based on the highest MLR amplitude, together with the highest ratio between the response at $\mathrm{BF}$ and an average response to stimuli other than BF. Such recordings were usually found in the central part of the MLR map. In these locations, multiple- unit activity was recorded between 100 and 1,200 $\mu \mathrm{m}$ beneath the surface using a commercial 16-channel electrode probe (Neuronexus, Ann Arbor, USA). The stimulation consisted of $60 \mathrm{~ms}$ tone pips at $0.5-45 \mathrm{kHz}$ frequency (4 steps per octave) and -10 to $+60 \mathrm{~dB}$ SPL intensity in $10 \mathrm{~dB}$ steps. The response was defined as a total spike count in the interval $10-70 \mathrm{~ms}$ after the stimulus onset, an average of 5 stimulations was calculated. Figure 2C shows examples of neuronal receptive fields (i.e. frequency-intensity plots with the response magnitude coded as shades of grey) compared with bar graph representation of MLR amplitudes obtained in the same location. Two frequency parameters were derived from the multiple-unit data for comparison to MLR-based maps; 1) the frequency at which the lowest stimulus intensity is required to evoke a response (characteristic frequency, $\mathrm{CF}$ ) and 2) the best frequency (BF) at $50 \mathrm{~dB}$ SPL, defined as the frequency of the stimulus that resulted in the highest response at this stimulation intensity. Figure 3B shows the correlation between BF of MLRs and BFs and CFs of 115 nearby recorded neurons. Approximately half (48.9\%) of the neuronal BFs fell into a half-octave range around the BF of the local MLR and $78 \%$ of the neuronal BFs were within one octave distance from its respective $\mathrm{BF}$ of MLR. Nevertheless, there were also around 3.5\% of neurons that showed a BF more than two octaves apart from the $\mathrm{BF}$ of the corresponding MLR. Values of neuronal CFs were higher compared to their respective $\mathrm{BF}$, especially in low-frequency tuned areas, which reflects a typical property of excitation in the AI (Tao et al. 2017), see examples 1, 3 and 7 in Figure 2C.

Certainly, there are several other methods for estimation of the functional organization of the cortex, usually based on optical techniques (Bakin et al. 1996, Grinvald and Hildesheim 2004, Issa et al. 2014) that provide more detailed maps including the secondary regions. However, these techniques require highly specialized devices and methods of analysis. Inspecting large portions of the auditory cortex by multi-electrode arrays has been demonstrated previously, using either commercially available products (Barth and Di 1990, Shiramatsu et al. 2016) or custom-designed arrays (Escabí et al. 2014, Insanally et al. 2016) in rats, ferrets (Owens et al. 1995) or cats (Fallon et al. 2016). The space resolution of our manually fabricated array ( 2 electrodes per $1 \mathrm{~mm}$ ) matches these machine-produced devices (inter-electrode distances of 200-800 $\mu \mathrm{m}$ ). We see a major advantage of the arrays based on flexible 
printed circuit boards (Insanally et al. 2016) or thin polyimide films (Owens et al. 1995, Escabí et al. 2014, Fallon et al. 2016) in that these are flexible, easily conform to the cortex surface, are less bulky than rigid arrays and potentially less irritating to the brain tissue during placement. In addition, the polyimide layer may be transparent. This feature, currently missing from our epoxy-resin array, was substituted by use of a sham transparent array. A flexible and thin body makes the polyimide film arrays especially suitable for chronic implantation, which, nevertheless, was not the aim of this study.

In conclusion, the electrode array described here proved to be a useful tool for fast mapping of the AI tonotopy, although it could not be used for detection of the secondary auditory fields. It may be fabricated in $24 \mathrm{~h}$ using common laboratory equipment and for negligible cost. The design can be adapted according to the actual needs, e.g. the electrode distribution might be based on equilateral triangles instead of the rectangular matrix, which would better fit into the round corners of the craniotomy. The number of channels can also be modified to meet the individual demands of the data acquisition system. Using a short stimulation protocol and simple analysis, it provides useful results within 20 minutes and makes the subsequent single- or multiple-unit recording session substantially more effective.

\section{Conflict of Interest}

There is no conflict of interest.

\section{Acknowledgements}

Results of the LQ1604 project were obtained with the financial contribution from the targeted support scheme of the National Sustainability Program II by the Czech Ministry of Education, Youth and Sports.

\section{References}

BAKIN JS, KWON MC, MASINO SA, WEINBERGER NM, FROSTIG RD: Suprathreshold auditory cortex activation visualized by intrinsic signal optical imaging. Cereb Cortex 6: 120-130, 1996.

BARTH DS, DI S: Three-dimensional analysis of auditory-evoked potentials in rat neocortex. J Neurophysiol 64: 1527-1536, 1990.

DORON NN, LEDOUX JE, SEMPLE MN: Redefining the tonotopic core of rat auditory cortex: physiological evidence for a posterior field. J Comp Neurol 453: 345-360, 2002.

ESCABÍ MA, READ HL, VIVENTI J, KIM D-H, HIGGINS NC, STORACE DA, LIU ASK, GIFFORD AM, BURKE JF, CAMPISI M, KIM Y-S, AVRIN AE, VAN DER SPIEGEL J, HUANG Y, LI M, WU J, ROGERS JA, LITT B, COHEN YE: A high-density, high-channel count, multiplexed $\mu \mathrm{ECoG}$ array for auditory-cortex recordings. J Neurophysiol 112: 1566-1583, 2014.

FALLON JB, IRVING S, PANNU SS, TOOKER AC, WISE AK, SHEPHERD RK, IRVINE DRF: Second spatial derivative analysis of cortical surface potentials recorded in cat primary auditory cortex using thin film surface arrays: comparisons with multi-unit data. J Neurosci Methods 267: 14-20, 2016.

GRINVALD A, HILDESHEIM R: VSDI: a new era in functional imaging of cortical dynamics. Nat Rev Neurosci 5: 874-885, 2004.

INSANALLY M, TRUMPIS M, WANG C, CHIANG C-H, WOODS V, PALOPOLI-TROJANI K, BOSSI S, FROEMKE RC, VIVENTI J: A low-cost, multiplexed ECoG system for high-density recordings in freely moving rodents. J Neural Eng 13: 026030, 2016.

ISSA JB, HAEFFELE BD, AGARWAL A, BERGLES DE, YOUNG ED, YUE DT: Multiscale optical Ca ${ }^{2+}$ imaging of tonal organization in mouse auditory cortex. Neuron 83: 944-959, 2014.

KALATSKY VA, POLLEY, DB, MERZENICH MM, SCHREINER CE, STRYKER MP: Fine functional organization of auditory cortex revealed by Fourier optical imaging. Proc Natl Acad Sci U S A 102: 13325-13330, 2005.

OHL FW, SCHEICH H, FREEMAN WJ: Topographic analysis of epidural pure-tone-evoked potentials in gerbil auditory cortex. $J$ Neurophysiol 83: 3123-3132, 2000.

OWENS AL, DENISON TJ, VERSNEL H, REBBERT M, PECKERAR M, SHAMMA SA: Multi-electrode array for measuring evoked potentials from surface of ferret primary auditory cortex. J Neurosci Methods 58: 209-220, 1995. 
PANDYA PK, RATHBUN DL, MOUCHA R, ENGINEER ND, KILGARD MP: Spectral and temporal processing in rat posterior auditory cortex. Cereb Cortex 18: 301-314, 2008.

PROFANT O, BURIANOVÁ J, SYKA J: The response properties of neurons in different fields of the auditory cortex in the rat. Hear Res 296: 51-59, 2013.

RUTKOWSKI RG, MIASNIKOV AA, WEINBERGER NM: Characterisation of multiple physiological fields within the anatomical core of rat auditory cortex. Hear Res 181: 116-130, 2003.

SALLY SL, KELLY JB: Organization of auditory cortex in the albino rat: sound frequency. J Neurophysiol 59: 1627-1638, 1988.

SHIRAMATSU TI, NODA T, AKUTSU K, TAKAHASHI H: Tonotopic and field-specific representation of longlasting sustained activity in rat auditory cortex. Front Neural Circuits 10: 59, 2016.

TAKAHASHI H, NAKAO M, KAGA K: Interfield differences in intensity and frequency representation of evoked potentials in rat auditory cortex. Hear Res 210: 9-23, 2005.

TAO C, ZHANG G, ZHOU C, WANG L, YAN S, ZHOU Y, XIONG Y: Bidirectional shifting effects of the sound intensity on the best frequency in the rat auditory cortex. Sci Rep 7: 44493, 2017.

THOMAS H, TILLEIN J: Functional organization of the auditory cortex in a native Chilean rodent (Octodon degus). Biol Res 30: 137-148, 1997. 